\title{
Gauss-Seidel limb scattering (GSLS) radiative transfer model development in support of the Ozone Mapping and Profiler Suite (OMPS) limb profiler mission
}

\author{
R. Loughman ${ }^{1}$, D. Flittner ${ }^{2}$, E. Nyaku ${ }^{1}$, and P. K. Bhartia ${ }^{3}$ \\ ${ }^{1}$ Department of Atmospheric and Planetary Sciences, Hampton University, Hampton, Virginia, USA \\ ${ }^{2}$ Science Directorate, NASA Langley Research Center, Hampton, Virginia, USA \\ ${ }^{3}$ Atmospheric Chemistry and Dynamics Laboratory, NASA Goddard Space Flight Center, \\ Greenbelt, Maryland, USA
}

Correspondence to: R. Loughman (robert.loughman@hamptonu.edu)

Received: 24 June 2014 - Published in Atmos. Chem. Phys. Discuss.: 28 July 2014

Revised: 27 December 2014 - Accepted: 4 February 2015 - Published: 17 March 2015

\begin{abstract}
The Gauss-Seidel limb scattering (GSLS) radiative transfer (RT) model simulates the transfer of solar radiation through the atmosphere and is imbedded in the retrieval algorithm used to process data from the Ozone Mapping and Profiler Suite (OMPS) limb profiler (LP), which was launched on the Suomi NPP satellite in October 2011. A previous version of this model has been compared with several other limb scattering RT models in previous studies, including Siro, MCC++, CDIPI, LIMBTRAN, SASKTRAN, VECTOR, and McSCIA. To address deficiencies in the GSLS radiance calculations revealed in earlier comparisons, several recent changes have been added that improve the accuracy and flexibility of the GSLS model, including
\end{abstract}

1. improved treatment of the variation of the extinction coefficient with altitude, both within atmospheric layers and above the nominal top of the atmosphere;

2. addition of multiple-scattering source function calculations at multiple solar zenith angles along the line of sight (LOS);

3. introduction of variable surface properties along the limb LOS, with minimal effort required to add variable atmospheric properties along the LOS as well;

4. addition of the ability to model multiple aerosol types within the model atmosphere.
The model improvements 1 and 2 are verified by comparison to previously published results (using standard radiance tables whenever possible), demonstrating significant improvement in cases for which previous versions of the GSLS model performed poorly. The single-scattered radiance errors that were as high as $4 \%$ in earlier studies are now generally reduced to $0.3 \%$, while total radiance errors generally decline from $10 \%$ to $1-3 \%$. In all cases, the tangent height dependence of the GSLS radiance error is greatly reduced.

\section{Introduction}

Incoming solar irradiance interacts with the Earth's atmosphere as it penetrates downwards towards the surface. The nature of these interactions depends upon the composition of the atmospheric layers, and this fact has been exploited by many successful remote sensing techniques that use observations of the radiation that exits the atmosphere to learn about atmospheric composition. Several viewing geometries have been exploited in this way, such as observing downwelling radiation at the Earth's surface, upwelling radiation at the top of the atmosphere (TOA), and the internal radiation field within the atmosphere. Upwelling radiance at the TOA along the line of sight (LOS) that passes near (but above) the horizon, and therefore does not intersect the Earth's surface, is commonly known as limb scattering (LS), as illustrated in 


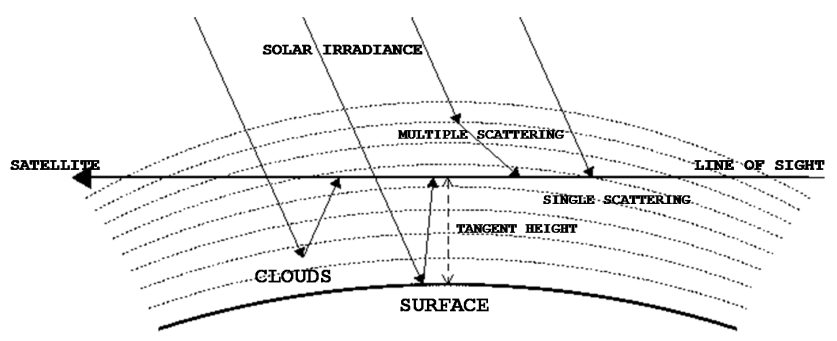

Figure 1. Illustration of the limb scattering viewing geometry (from Rault and Loughman, 2013).

Table 1. Rayleigh scattering cross section $\sigma_{R}$ and ozone cross section $\sigma_{O}$ used in comparisons to L04 results. These values are taken from Table 3 of L04.

\begin{tabular}{lcrr}
$\lambda(\mathrm{nm})$ & 325 & 345 & 600 \\
$\sigma_{R}\left(\mathrm{~cm}^{2}\right)$ & $4.022 \times 10^{-26}$ & $3.120 \times 10^{-26}$ & $3.167 \times 10^{-27}$ \\
$\sigma_{O}\left(\mathrm{~cm}^{2}\right)$ & $1.451 \times 10^{-20}$ & 0 & $5.21709 \times 10^{-21}$ \\
\hline
\end{tabular}

Fig. 1. LS observations have been successfully employed to infer the properties of the Earth's atmosphere in several past and present remote sensing missions, such as SME (Rusch et al., 1984; Mount et al., 1984), SOLSE/LORE (McPeters et al., 2000), OSIRIS (Llewellyn et al., 2004), SCIAMACHY (Bovensmann et al., 1999), SAGE III (Rault, 2005), GOMOS (Kyrölä et al., 2004), and Ozone Mapping and Profiler Suite (OMPS) limb profiler (LP) (Flynn et al., 2006).

An essential tool for interpretation of LS observations is a radiative transfer (RT) model capable of simulating the LS radiance field for a specified atmosphere. Many models have been developed to simulate LS radiances, including the DART (Whitney, 1977), Siro (Oikarinen et al., 1999), LIMBTRAN (Griffioen and Oikarinen, 2000), CDIPI (Rozanov et al., 2001), VECTOR (McLinden et al., 2002), MCC++ (Postylyakov, 2004), Gauss-Seidel limb scattering (GSLS) (Loughman et al., 2004), McSCIA (Spada et al., 2006), SASKTRAN (Bourassa et al., 2008), McArtim (Deutschmann et al., 2011), MOCRA (Premuda et al., 2012), and SCIATRAN (Rozanov et al., 2014) models. A previous paper (Loughman et al., 2004, called L04 herein) assesses the consistency of several LS RT models at an earlier stage in their development and has provided a useful benchmark for subsequent RT model development (Spada et al., 2006; Bourassa et al., 2008; McLinden and Bourassa, 2010). The L04 paper highlighted several shortcomings in the GSLS model, and improving the model by rectifying those shortcomings is the primary motivation for the present work.

\section{GSLS model overview}

The GSLS model is built from the previous models described by Herman et al. $(1994,1995)$ and is summarized in L04. The model atmosphere is specified by input pressure, tem- perature, absorbing gas number density, and aerosol extinction profiles (the profiles used in this study mimic the L04 profiles and are provided in the Supplement). The ozone and Rayleigh scattering cross sections also follow L04 and are given in Table 1. Refraction and molecular depolarization by molecular scattering are included as options in the GSLS model but are turned off in this study (to enable comparisons with L04 results). For L04 comparisons, the aerosol scattering properties are specified by the Henyey-Greenstein phase function, with single-scattering (SS) albedo $=1.0$ and asymmetry parameter $=0.7$. In later work (see Sect. 4.5), Mie theory is used to calculate the aerosol scattering phase functions.

The viewing geometry is specified by the solar zenith angle and relative azimuth angle at the tangent point (TP) for the LOS, denoted by $\theta_{T}$ and $\phi_{T}$, respectively. Radiances are calculated at several wavelengths $\lambda$ and tangent heights $h$. For single-scattering calculations, the solar beam attenuation is calculated to each point along the LOS, including the curvature of the spherical atmosphere as well as the variation of solar zenith angle and solar beam attenuation along the LOS. The attenuation of the scattered beam along the LOS is also calculated accounting for the curvature of the atmosphere. The multiple-scattering (MS) source function is calculated at one or more points along the LOS using the pseudo-spherical version of the RT model described by Herman et al. (1994, 1995). In the L04 GSLS model, the MS source functions were calculated only at the TP (solar zenith angle $=\theta_{T}$ ), illustrated by the point $T$ in Fig. 2. This has been updated to calculate the MS source functions at multiple solar zenith angles along the LOS in the present GSLS model, as described further in Appendix A.

The GSLS model has been used for retrieval applications on missions including SOLSE/LORE (Flittner et al., 2000), SAGE III (Rault, 2005; Rault and Taha, 2007), GOMOS (Taha et al., 2008), SCIAMACHY (Taha et al., 2011) and OMPS LP (Rault and Loughman, 2013). These retrieval algorithms have shown a remarkable degree of accuracy despite the shortcomings of the GSLS model, but development of a more accurate version of the GSLS model is desirable for the purpose of interpreting residuals (differences between measured radiances and radiances calculated for the desired model atmosphere). This paper is devoted to describing and demonstrating the improvements made to the GSLS model.

\section{OMPS instrument description}

The OMPS instruments were designed and built by Ball Aerospace and Technology Corporation. The suite includes three instruments: a nadir profiler instrument carrying on the Solar Backscatter Ultraviolet Radiometer measurement record, a nadir mapping instrument carrying on the Total Ozone Mapping Spectrometer/Ozone Monitoring Instrument measurement record, and the limb profiler instrument. OMPS LP measures the LS radiance by simultaneously imaging the 


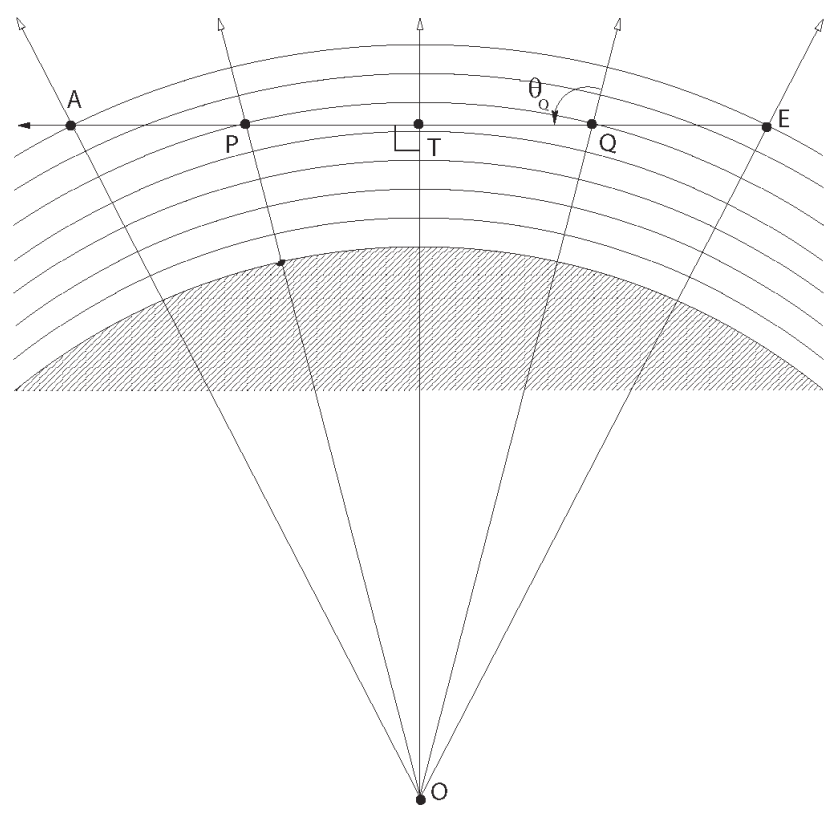

Figure 2. Illustration of the LS geometry. The radiation travels along the LS LOS from the TOA at point E downward to the tangent point $\mathrm{T}$, then upward to the TOA at A. Zeniths originate at the center of the spherical Earth $\mathrm{O}$ and radiate outward through the atmosphere (from Fig. 9 of Loughman et al., 2004).

entire vertical extent of the limb through three vertical slits that cover a vertical tangent height range of approximately $100 \mathrm{~km}$, with an instantaneous field of view that translates to approximately $1.3-1.7 \mathrm{~km}$ tangent height. The wavelength range is $290-1000 \mathrm{~nm}$, dispersed by a prism that provides high spectral resolution (1-2 nm) in the UV but lower spectral resolution at longer wavelengths $(11 \mathrm{~nm}$ at $600 \mathrm{~nm}$ and $31 \mathrm{~nm}$ at $1000 \mathrm{~nm}$ ).

As the name implies, the OMPS instrument suite was designed primarily to measure ozone column amount and profile information. The OMPS LP instrument also has significant sensitivity to other species (such as molecular number density, $\mathrm{NO}_{2}$, and aerosols), but the relatively low spectral resolution is not optimal for $\mathrm{NO}_{2}$ retrievals, and the radiance characterization must be improved to take full advantage of the aerosol sensitivity of the measurements (for example, reducing the residual stray light at $1000 \mathrm{~nm}$ to a level significantly below its current $10 \%$ level) (Jaross et al., 2014).

The first OMPS mission was mounted on the Suomi NPP satellite, which launched on 28 October 2011. Suomi NPP is in a near-circular, sun-synchronous orbit, with a 01:30 p.m. local equator crossing ascending node and a mean altitude of $833 \mathrm{~km}$. The center slit of OMPS LP is aligned with the satellite ground track, with the other two slits aimed $4.25^{\circ}$ on either side. This orbit dictates the viewing geometry of the OMPS LP measurements; in particular, the single-scattering angle at the TP $\Theta_{T}$ is largest (approaching $160^{\circ}$ ) as the sunlit portion of each orbit begins in the Southern Hemisphere and smallest (approaching $20^{\circ}$ ) at the end of the sunlit portion in the Northern Hemisphere. For more comprehensive information concerning the OMPS LP sensor, consult Flynn et al. (2006), Rault and Loughman (2013), and Jaross et al. (2014).

\section{GSLS model improvements}

\subsection{Optical path lengths}

Siro is a Monte Carlo model that accounts for all of the complications associated with RT in the limb of a spherical atmosphere, and it therefore served as the benchmark in the L04 radiance comparison study. That study notes a bias in the GSLS SS radiances relative to the Siro SS radiances, arising from the approximation used to calculate the limb optical path length $\tau$ through a layer in the L04 version of GSLS. The correct calculation of $\tau$ requires integrating the extinction coefficient $\beta$ along the LOS through the relevant layer.

$\tau=\int_{0}^{s} \beta \mathrm{d} s^{\prime}$

In both L04 and the current study, the influence of refraction is neglected, so it is appropriate to refer to the path taken by a photon as a straight line, with the geometric path length coordinate $s^{\prime}$ increasing from 0 to $s$ as the photon traverses the layer. The model atmosphere is defined by specifying the value of $\beta$ at discrete altitudes within the model atmosphere (by specifying values from which $\beta$ can be derived, such as the number densities and cross sections for various sources of extinction). In both L04 and this study, the model atmosphere consists of 100 layers, each $1 \mathrm{~km}$ thick, with the top of the atmosphere $100 \mathrm{~km}$ above the Earth's surface (the significance of the top of the atmosphere in the RT model is discussed further in Sect. 4.4).

This atmospheric definition provided each model in the L04 comparison with consistent values of $\beta$ at the top $\left(\beta_{t}\right)$ and bottom $\left(\beta_{b}\right)$ of each layer but allowed the various RT models to make different assumptions about how $\beta$ should vary within the atmospheric layers. In the L04 version of GSLS, the average value of $\beta$ within the layer is multiplied by the layer geometric path length $s$ to determine $\tau$ in all layers except the tangent layer; that is,

$\tau=\frac{s\left(\beta_{\mathrm{t}}+\beta_{\mathrm{b}}\right)}{2}$.

As noted in L04, the Siro model makes a more accurate approximation by assuming that $\beta$ varies as a linear function of altitude within each layer. Figure 4 of L04 shows that this assumption has a significant impact on the LS radiance calculation, particularly for the single-scattered radiance: the SS radiances calculated by the L04 GSLS model agree well with the SS radiances produced by other models that use the approximation given in Eq. (2) and also agree with Siro SS ra- 
Table 2. Cases for which the L04 and current GSLS radiances are compared to Siro radiances.

\begin{tabular}{lc}
\hline$\lambda(\mathrm{nm})$ & $325,345,600 \mathrm{~nm}$ \\
$h(\mathrm{~km})$ & $10-60 \mathrm{~km}(5 \mathrm{~km} \mathrm{steps})$ \\
$\theta_{T}\left(^{\circ}\right)$ & $15,60,80,90$ \\
$\phi_{T}\left(^{\circ}\right)$ & $20,90,160$ \\
\hline
\end{tabular}

diances for tangent height $h \approx 10 \mathrm{~km}$. However, the SS radiances calculated by models that use Eq. (2) diverge from the Siro SS radiances as $h$ increases, disagreeing by as much as $1 \%$ for $h=60 \mathrm{~km}$. When the Siro model was modified to calculate $\tau$ as shown in Eq. (2), the SS radiance differences were greatly reduced.

Figures $3 \mathrm{a}$ and $4 \mathrm{a}$ collect information shown in Figs. 6-8 of L04 in a different format, showing the difference between the L04 GSLS SS radiances and the Siro SS radiances for the cases listed in Table 2. Cases are specified by $\lambda, \theta_{T}$, and $\phi_{T}$. The differences in Figs. 3a and 4a range from $-2.6 \%$ to $+3.6 \%$. In Figs. $3 b$ and $4 b$, Siro SS radiances are instead compared to the current GSLS SS radiances. Implementation of the linear variation of $\beta$ with altitude in the current GSLS model both reduces the disagreement range and virtually eliminates the altitude dependence of the disagreement.

Another GSLS error was also discovered and corrected during the development of this paper. This error was related to the aerosol phase function, which is calculated twice in the GSLS model: once at the exact $\Theta_{T}$ value (appropriate for SS calculations) and again at $1^{\circ}$ increments in scattering angle (appropriate for MS calculations). In the L04 GSLS model, the latter aerosol phase function was used for SS calculations (using the gridded scattering angle nearest $\Theta_{T}$ ) rather than the former. The associated error in the total phase function value was small in most instances, but it led to significant radiance errors in the worst cases. The worst cases occurred when the value of $\Theta_{T}$ was near a half-integer, the aerosol phase function was changing rapidly with scattering angle, and the scattering was dominated by aerosols (i.e., the forward scattering region, at longer wavelengths). In the worst cases among the L04 tests $\left(\lambda=600 \mathrm{~nm}, \theta_{T}=60^{\circ}\right.$ or $80^{\circ}$, $\phi_{T}=20^{\circ}$ ), the resulting SS radiance error was as large as $1.5 \%$.

Figure $3 \mathrm{~b}$ illustrates that the GSLS SS radiances now agree with their Siro counterparts, with disagreement ranging from -0.02 to $+0.26 \%$. The agreement deteriorates slightly due to the large solar zenith angles for the cases shown in Fig. 4b, but remains in the range -0.025 to $+0.85 \%$. Excluding the $\theta_{T}=90^{\circ}$ cases reduces the range of disagreement to -0.025 to $+0.33 \%$.

The generally close agreement between Siro and current GSLS SS radiances is not surprising, since both models now use the same approximation for the variation of $\beta$ within atmospheric layers. The method used to compute $\tau$ when $\beta$ varies linearly with altitude in the current GSLS model is pre- a)

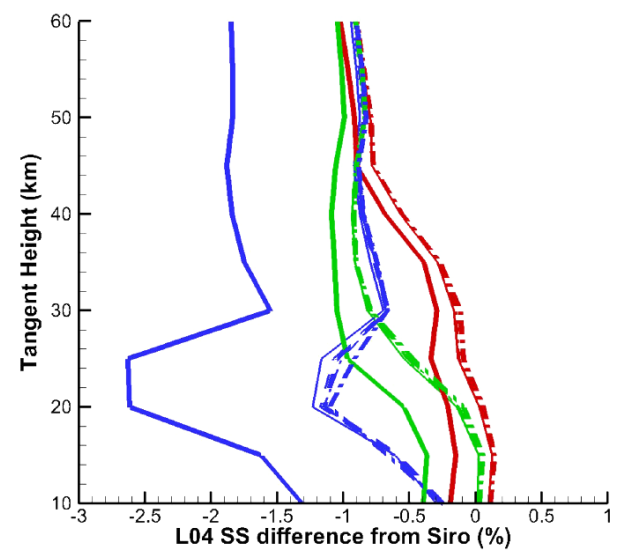

b)

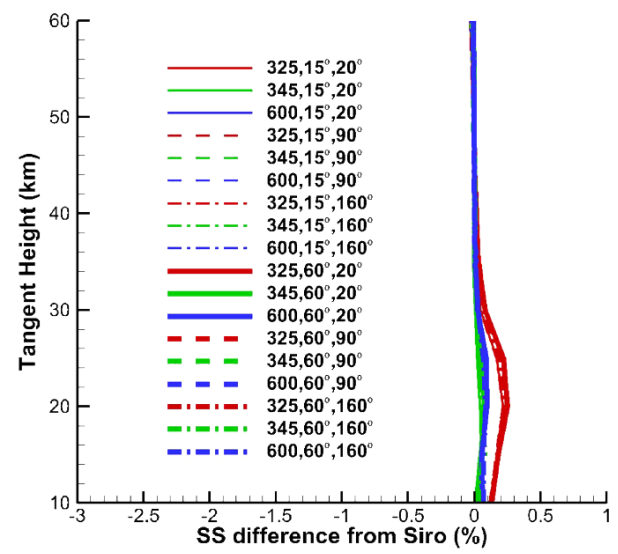

Figure 3. (a) Comparison of L04 GSLS SS radiances to Siro SS radiances for $\theta_{T}=15^{\circ}$ and $60^{\circ}$. (b) Comparison of current GSLS SS radiances to Siro SS radiances for the same cases. The figure legend indicates $\lambda(\mathrm{nm}), \theta_{T}\left({ }^{\circ}\right)$, and $\phi_{T}\left({ }^{\circ}\right)$. Agreement with Siro results is improved by allowing $\beta$ to vary linearly with altitude in the current GSLS model, as described further in Appendix B.

sented more fully in Appendix B. It is relatively straightforward, admitting an analytic solution and requiring only application of integral forms that are readily available in standard references. Its computation cost is therefore modest (producing a negligible increase in calculation time).

As shown in Figs. 3-4, implementing the linear variation of $\beta$ with altitude produced significant improvement in the SS radiance comparison. Similar modifications were also made to allow the GSLS model to compute layer path lengths the same way in the MS source function calculations as well. As noted in L04, the MS source functions are computed using a modified version of the "cone code" described in (Herman et al., 1994, 1995). Given the short path lengths between the zenith and conical boundary and the relatively small contribution of LS paths to the overall value of the MS source function, this modification produced a negligible change in the MS radiance calculation. 
a)

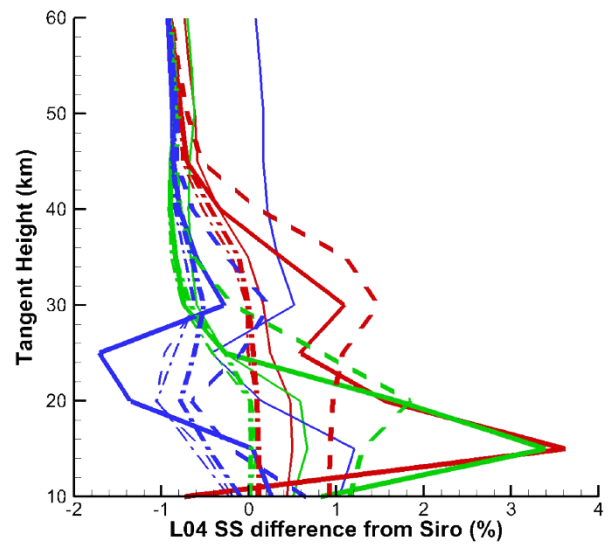

b)

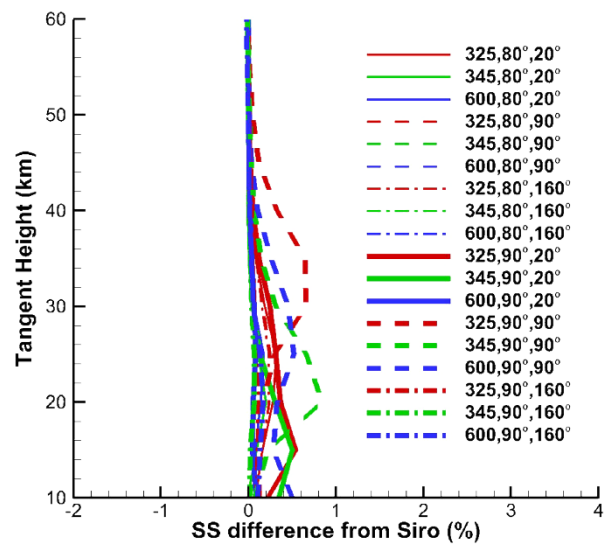

Figure 4. (a) Comparison of L04 GSLS SS radiances to Siro SS radiances for $\theta_{T}=80^{\circ}$ and $90^{\circ}$. (b) Comparison of current GSLS SS radiances to Siro SS radiances for the same cases. The figure legend indicates $\lambda(\mathrm{nm}), \theta_{T}\left({ }^{\circ}\right)$, and $\phi_{T}\left(^{\circ}\right)$. Agreement with Siro results is improved by allowing $\beta$ to vary linearly with altitude in the current GSLS model, as described further in Appendix B.

\subsection{MS solar zenith angles}

As described in Appendix A of L04, the L04 GSLS model computes the MS source function for the solar zenith angle at TP $\left(\theta_{T}\right)$ for the LOS of interest (see $\overrightarrow{O T}$ in Fig. 2). This approximation contributes to the speed of the L04 GSLS model at the cost of lost accuracy for some MS radiance calculations in which the MS source function varies significantly along the LOS. The total scattered (TS) radiances $(\mathrm{TS}=\mathrm{SS}+\mathrm{MS}$ ) calculated by the L04 GSLS model differ from the Siro reference radiances by as much as $12 \%$ for surface reflectivity $R=0.95$ and $\theta_{T} \leq 80^{\circ}$, as shown in Fig. 5a (which displays information taken from the Figs. 6-8 of L04 in modified form). For cases when the LOS is optically thin (such as Fig. 6a), the radiance is dominated by contributions from the layers near the TP, so use of the MS source function values calculated at the TP for the entire LOS integration causes little error. However, the L04 GSLS radiances differ most from a)

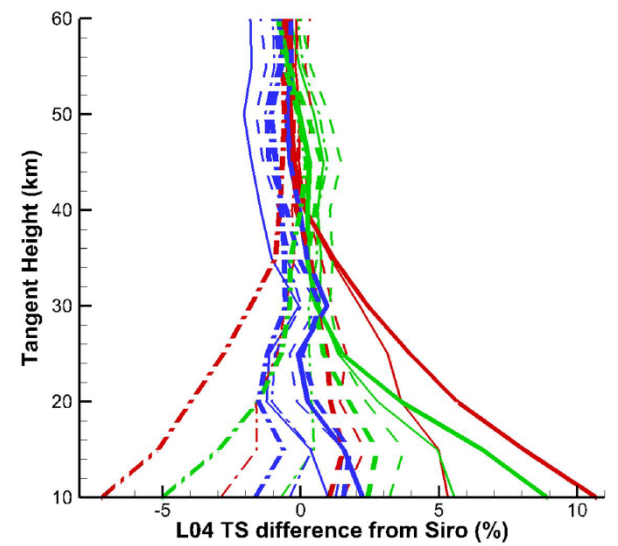

b)

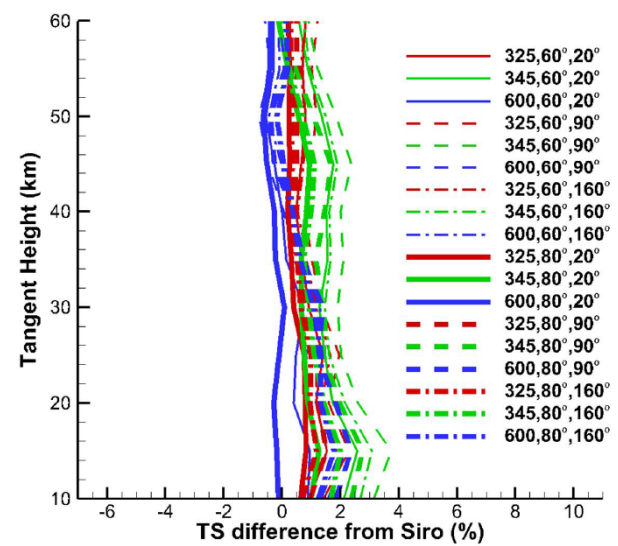

Figure 5. (a) Comparison of L04 GSLS TS radiances to Siro TS radiances when $R=0.95$, at $\theta_{T}=60^{\circ}$ and $80^{\circ}$. (b) Comparison of current GSLS TS radiances to Siro TS radiances for the same cases. The figure legend indicates $\lambda(\mathrm{nm}), \theta_{T}\left({ }^{\circ}\right)$, and $\phi_{T}\left(^{\circ}\right)$. Agreement with Siro results is improved by the introduction of calculations with $N_{\theta}>1$ in the current GSLS model, as described further in Sect. 4.2 and Appendix A.

the Siro radiances for ultraviolet (UV) wavelengths (325 and $345 \mathrm{~nm})$ at small tangent heights $(h<30 \mathrm{~km})$, where the LOS optical path becomes large and contributions from the layers between the TP and the observer become more significant, as illustrated in Fig. $6 \mathrm{~b}$ and c. Note that the current GSLS model uses pseudo-spherical approximations that prevent it from calculating MS source functions properly when the sun is at or below the horizon, so cases that require such calculations will not be analyzed in this study.

The current GSLS model has been modified to allow computation of MS source functions at several solar zenith angles along the LOS (e.g., $\overrightarrow{O A}, \overrightarrow{O P}, \overrightarrow{O T}, \overrightarrow{O Q}, \overrightarrow{O E}$ in Fig. 2). This modification allows better representation of the MS source function, as shown by the improved total scattered radiance agreement with Siro in Fig. 5b. The cases shown in Fig. 5a for L04 GSLS radiances are repeated in Fig. $5 \mathrm{~b}$ for current GSLS radiances, for which TS radiance differences relative to Siro 
a)

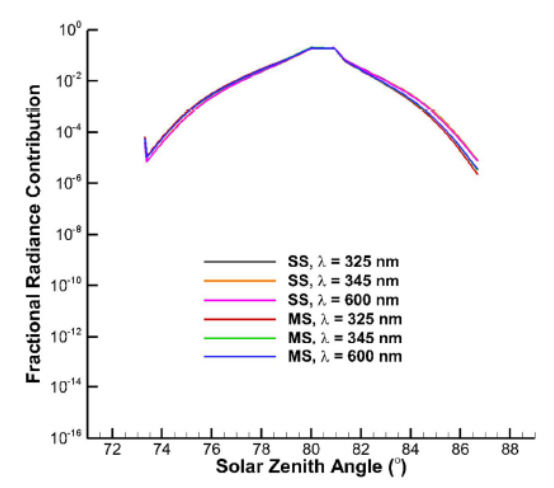

b)

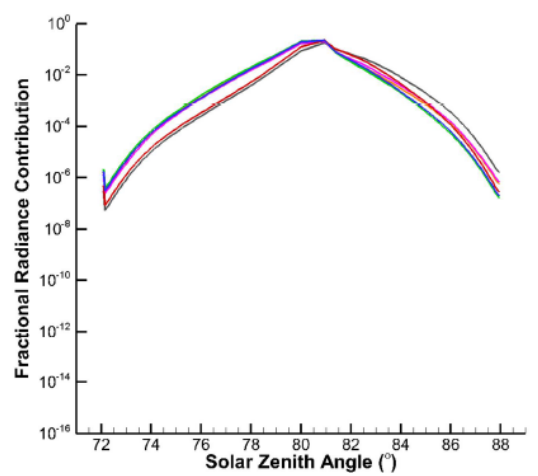

c)

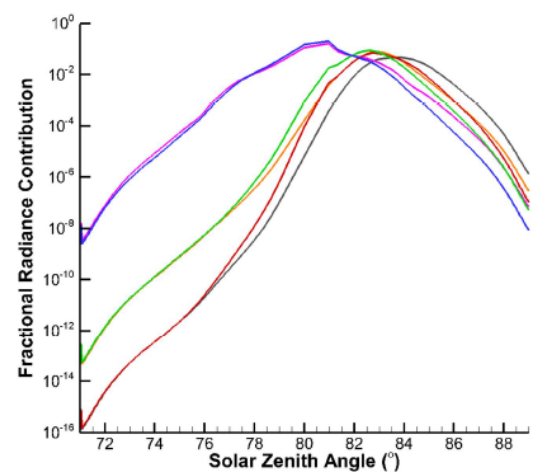

Figure 6. Relative contribution for the layers along the LOS for $\theta_{T}=80^{\circ}, \phi_{T}=20^{\circ}, R=0.95$, and $h=50 \mathrm{~km}(\mathbf{a}), h=30 \mathrm{~km}(\mathbf{b})$ and $h=10 \mathrm{~km}(\mathbf{c})$. The legend indicates whether the SS or MS source function is shown, followed by $\lambda(\mathrm{nm})$.

Table 3. Cases studied to determine the appropriate number of MS solar zenith angles $N_{\theta}$ to be used in the GSLS model.

\begin{tabular}{lc}
\hline$\lambda(\mathrm{nm})$ & $325,345,600 \mathrm{~nm}$ \\
$h(\mathrm{~km})$ & $10-60 \mathrm{~km}(5 \mathrm{~km} \mathrm{steps})$ \\
$\theta_{T}\left(^{\circ}\right)$ & $15,45,60,70,80,85$ \\
$\phi_{T}\left(^{\circ}\right)$ & $0,20,40,60,80,90,100,120,140,160,180$ \\
\hline
\end{tabular}

are reduced to $\leq 4 \%$. The other LS RT models compared to Siro earlier either already contain a multi-zenith capability for MS source function calculations (e.g., CDIPI, VECTOR, LIMBTRAN, and SASKTRAN) or explicitly simulate each photon, eliminating the need for this capability (e.g., Siro, $\mathrm{MCC}++$, and McSCIA).

A limited study was done to assess the required number and placement of the MS solar zenith angles along the LOS to reach a given standard of accuracy. In this study, the scene reflectivity $R$ was set to 0.95 (to maximize the impact of MS on the TS radiance). The viewing geometry was varied as described in Table 3. As a reference, the current GSLS RT model was run with the number of MS solar zenith angles $N_{\theta}=143$. First, a MS solar zenith angle was placed at each point for which the LOS at $h=30 \mathrm{~km}$ intersects an atmospheric level. This yields $141 \mathrm{MS}$ solar zenith angles, with the remaining $2 \mathrm{MS}$ solar zenith angles set where the LOS with $h=0 \mathrm{~km}$ intersects the TOA at each end of the LOS. These reference calculations were then compared to the results obtained with $N_{\theta}=1$ (MS solar zenith angle $=\theta_{T}$ ). For the purpose of this study, additional MS solar zenith angles were gradually added until the difference from the reference calculation (with 143 MS solar zenith angles) became $<0.2 \%$ at all values of $h$ and $\lambda$.

Placement of the additional MS solar zenith angles was motivated by Fig. 6, which demonstrates how the layers that contribute most to the radiance shift from the TP towards the observer as $h$ decreases, particularly for the optically thicker UV wavelengths considered $(325,345 \mathrm{~nm})$. The value of $N_{\theta}$ that is required to meet the $0.2 \%$ difference standard is given in Table 4 and is color-coded in Fig. 7. The sequence and placement of the MS solar zenith angles were as follows:

1. solar zenith angle at the zenith that intersects the LOS at the TP (i.e., $\overrightarrow{O A}$ in Fig. 2);

2. solar zenith angle at the zenith that intersects the LOS at the TOA on the "near side" (zenith lies between the TP and observer, i.e., $\overrightarrow{O A}$ in Fig. 2);

3. solar zenith angle at the zenith that intersects the LOS at the TOA on the "far side" (for which the TP is between the zenith and observer, i.e., $\overrightarrow{O E}$ in Fig. 2);

4. solar zenith angle at the zenith that intersects the LOS at the level immediately above the TP on the "near side" (i.e., $\overrightarrow{O P}$ in Fig. 2);

5. solar zenith angle at the zenith that intersects the LOS at the level immediately above the TP on the "far side" (i.e., $\overrightarrow{O Q}$ in Fig. 2);

6. another solar zenith angle added on the "near side" of the LOS, placed by experimentation with the cases listed in Table 3). Best results were obtained when the angle between the TP zenith ( $\overrightarrow{O T}$ in Fig. 2$)$ and the new zenith $=2.86159^{\circ}$.

The SS radiance is unaffected by the number or placement of the MS solar zenith angles, and the change in TS radiance due to increasing $N_{\theta}$ is slight when $\theta_{T} \approx 0^{\circ}$ (sun nearly overhead at the TP) or $\phi_{T} \approx 90^{\circ}$ (LOS nearly perpendicular to the solar plane) because those conditions minimize the variation of the solar illumination along the LOS. As the sun 


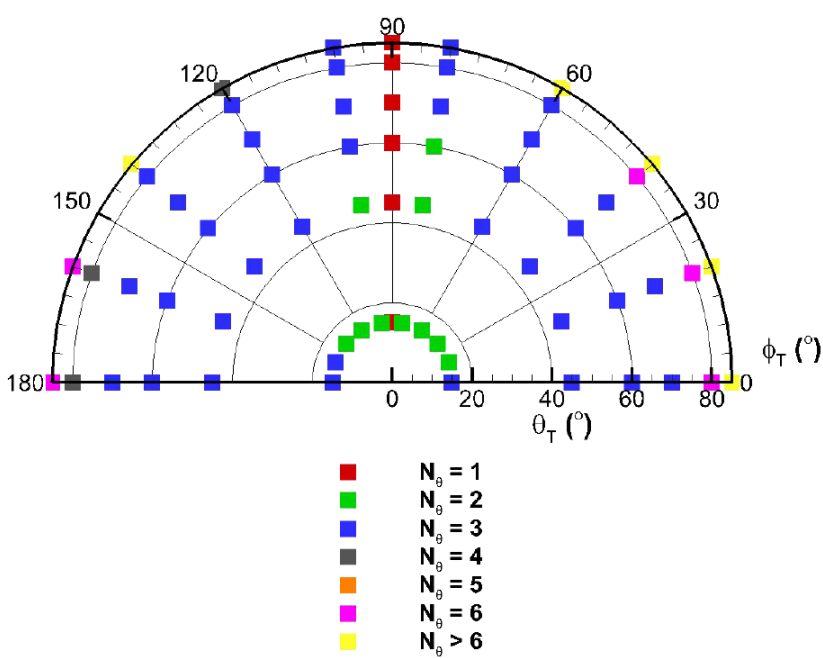

Figure 7. The number of MS solar zenith angles $N_{\theta}$ needed to cause the calculated GSLS TS radiance to agree with the reference calculations (which use $N_{\theta}=143$ ) to within $0.2 \%$ at all $\lambda$ and $h$ when $R=0.95$. This analysis is illustrated for each of the $\theta_{T}$ and $\phi_{T}$ values listed in Table 3, and its results are shown in table form in Table 4.

approaches the horizon or the LOS becomes more aligned with the solar plane, the importance of increasing $N_{\theta}$ grows. Comparing Fig. 5a to 5b shows that the radiances at small tangent heights ( $h=10 \mathrm{~km}$ is the smallest included in this analysis) are most affected by the number and placement of the MS solar zenith angles. The UV wavelengths (for which the atmosphere is optically thicker) are also most sensitive to the details of MS solar zenith angle placement.

As shown in Table 4 and Fig. 7, the L04 GSLS model (for which $N_{\theta}=1$ ) meets the standard of TS radiance accuracy better than $0.2 \%$ only for cases with $\phi_{T}=90^{\circ}$. However, $N_{\theta}=2$ is sufficient for some viewing geometries with small $\theta_{T}$ and/or $\phi_{T}$ near $90^{\circ}$, and $N_{\theta}=3$ suffices for a large range of viewing conditions. Adding the fourth and fifth MS solar zenith angles produces marginal improvement, but $N_{\theta}=6$ suffices for nearly every remaining case. The cases that require $N_{\theta}>6$ to meet the criterion of TS radiance accuracy better than $0.2 \%$ arise at UV wavelengths with $\theta_{T}=85^{\circ}$ and $\phi_{T}$ far from $90^{\circ}$. In such cases, part of the LOS is often in shadow, and therefore the current GSLS model performance cannot be trusted in any case. The question of how many MS solar zenith angles are necessary under such "twilight" conditions is not addressed by the present study.

Increasing $N_{\theta}$ clearly increases the computational effort of the calculation, so the trade-off between accuracy and runtime must be carefully considered for a given application. For example, a GSLS run for which $N_{\theta}=6$ (the maximum required for the cases studied) takes roughly twice as long as a GSLS run with $N_{\theta}=2$ (the minimum required for any case with $\phi_{T} \neq 90^{\circ}$ ). Also note that the GSLS modifications described in this section make it easy to vary the surface re-
Table 4. The number of MS solar zenith angles $N_{\theta}$ needed to cause the calculated GSLS TS radiance to agree with the reference calculations (which use $N_{\theta}=143$ ) to within $0.2 \%$ at all $\lambda$ and $h$ when $R=0.95$. This analysis was done for each of the $\theta_{T}$ and $\phi_{T}$ values listed in Table 3, and its results are also illustrated in Fig. 7.

\begin{tabular}{lrrrrrr}
\hline$\theta_{T}=$ & $15^{\circ}$ & $45^{\circ}$ & $60^{\circ}$ & $70^{\circ}$ & $80^{\circ}$ & $85^{\circ}$ \\
$\phi_{T}=0^{\circ}$ & 3 & 3 & 3 & 3 & 6 & $>6$ \\
$\phi_{T}=20^{\circ}$ & 2 & 3 & 3 & 3 & 6 & $>6$ \\
$\phi_{T}=40^{\circ}$ & 2 & 3 & 3 & 3 & 6 & $>6$ \\
$\phi_{T}=60^{\circ}$ & 2 & 3 & 3 & 3 & 3 & $>6$ \\
$\phi_{T}=80^{\circ}$ & 2 & 2 & 2 & 3 & 3 & 3 \\
$\phi_{T}=90^{\circ}$ & 1 & 1 & 1 & 1 & 1 & 1 \\
$\phi_{T}=100^{\circ}$ & 2 & 2 & 3 & 3 & 3 & 3 \\
$\phi_{T}=120^{\circ}$ & 2 & 3 & 3 & 3 & 3 & 4 \\
$\phi_{T}=140^{\circ}$ & 2 & 3 & 3 & 3 & 3 & $>6$ \\
$\phi_{T}=160^{\circ}$ & 3 & 3 & 3 & 3 & 4 & $>6$ \\
$\phi_{T}=180^{\circ}$ & 3 & 3 & 3 & 3 & 4 & $>6$ \\
\hline
\end{tabular}

Table 5. Cases used to verify the GSLS polarized radiance by comparison to the results of Natraj et al. (2009). The cases are identified by the cosine of the solar zenith angle $\mu_{0}$, the Lambertian surface reflectivity $R$, and the Rayleigh scattering optical depth $\tau_{R}$.

\begin{tabular}{lc}
\hline$\mu_{0}$ & $0.2,1.0$ \\
$R$ & $0,0.8$ \\
$\tau_{R}$ & $0.5,1.0$ \\
\hline
\end{tabular}

flection properties along the LOS. With minor code modifications, variation of the atmosphere along the LOS could also be added.

Finally, it must be noted that the current GSLS TS radiances uniformly exceed the Siro TS values by a small amount (1-3\%) for the cases shown in Fig. 5b. Some brief experiments (not included in this paper) indicate that the observed overestimate increases with increasing $R$ and with decreasing $\theta_{T}$, clearly suggesting that the treatment of radiance reflected by the surface that underlies the model atmosphere is a possible source of error. The reason for this discrepancy is unknown, but it may be related to the fact that RT models using flat (or pseudo-spherical, like the current GSLS) atmospheres for MS calculations tend to overestimate upwelling radiation, as noted by McLinden and Bourassa (2010).

\subsection{Influence of polarization}

The correct vector RT equation includes the influence of polarization on the scattered radiation field by representing the scattering process with $4 \times 4$ phase matrices. This produces the four-element Stokes parameter vector, which includes the radiance and all relevant phase information for each scattered radiance value. The scalar RT equation neglects the influence of polarization on the scattered radiance, replacing the $4 \times 4$ scattering phase matrices with scalar phase functions and producing scalar radiances. This approximation has no 


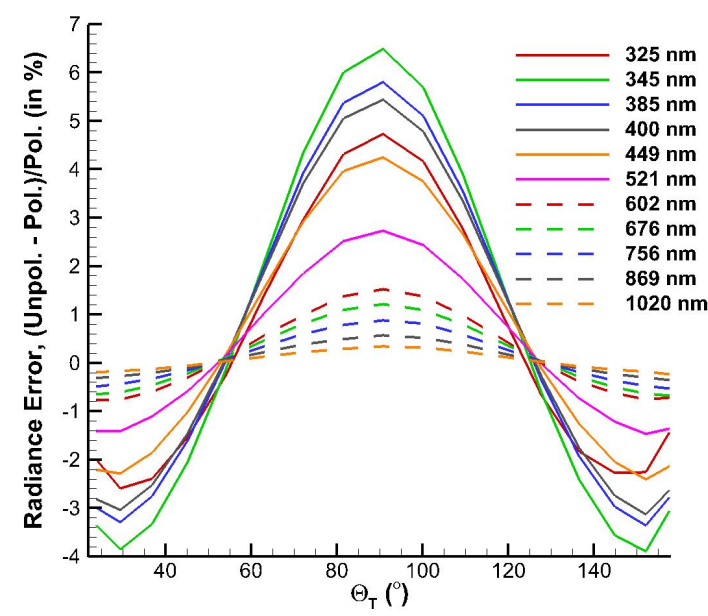

Figure 8. The TS radiance error that results from neglecting polarization is plotted as a function of SS angle $\Theta_{T}$ for a series of radiance simulations that employ the viewing geometry of a simulated NPP OMPS LP orbit. The tangent height $h=40 \mathrm{~km}, R=0$, and the legend indicates $\lambda(\mathrm{nm})$.

clear theoretical basis and produces scalar radiances that may differ from their vector counterparts by $>12 \%$ (Mishchenko et al., 1994), but the scalar approximation to the RT equation nonetheless is frequently used. Several LS radiance retrieval algorithms (including the OMPS LP algorithms) use scalar RT models, but the scalar radiance error is not generally a significant source of retrieval error, primarily due to the tangent height normalization of the radiances used for retrievals (Loughman et al., 2005). The vector GSLS model is valuable primarily for tasks such as directly interpreting the residuals between the measured OMPS LP radiances and the GSLS calculated radiances.

The accuracy of the vector radiances calculated by the current GSLS model was verified in two ways. First, we confirmed that the current GSLS model duplicates the L04 GSLS polarized radiances shown in Fig. 3 of Loughman et al. (2004) to the $0.01 \%$ level, provided that the optical path lengths were calculated as shown in Eq. (2). The second set of comparisons involved the results tabulated by Natraj et al. (2009), which represent an updated version of the wellknown Rayleigh scattering calculations initially produced by Coulson et al. (1960). The Natraj et al. (2009) comparisons cover the cases listed in Table 5. To enable this comparison, the current GSLS model was run as a "flat atmosphere" model (by increasing the Earth's radius by a factor of 1000). The emerging radiances at the TOA and the surface match the Natraj et al. (2009) results to better than $1.4 \%$ for all LOS, with agreement better than $0.5 \%$ except in a few cases.

The scalar LS radiances differ significantly from correct polarized radiances by as much as -4 to $+7 \%$, as shown in Fig. 8. The overall behavior of these curves follows the expected pattern (Mishchenko et al., 1994), with the largest errors appearing at $345 \mathrm{~nm}$, when (1) Rayleigh scattering dominates, (2) just a few scattering events are likely for a typical photon (atmospheric optical depth is $\approx 1$ ), (3) atmospheric absorption is weak, and (4) surface reflectivity $R$ is small. The error is greatest when $\Theta_{T} \approx 90^{\circ}$, which occurs near the midpoint of the NPP OMPS LP orbit (in the tropics). As noted in L04, the SS angle $\Theta_{T}$ is related to the solar zenith angle and relative azimuth angle at the $\operatorname{TP}\left(\theta_{T}\right.$ and $\phi_{T}$, respectively) by

$\cos \Theta_{\mathrm{T}}=\sin \theta_{\mathrm{T}} \cos \phi_{\mathrm{T}}$.

\subsection{Chapman layer}

The TOA for the L04 GSLS model atmosphere was mentioned in Sect. 4.1. Of course, a real atmosphere does not abruptly end at any particular height but instead becomes more and more rarefied as height increases (consistent with hydrostatic balance in the long-term average). The RT model atmosphere is typically specified (by profiles of pressure, temperature, aerosols, absorbing gases, etc.) to some notional TOA altitude. The altitude at which the TOA can be placed depends on the application for which the RT calculations are done, which determines the required accuracy of the radiances. The atmosphere above the TOA can be treated in several ways, which also influences the necessary TOA for a particular application. The simplest approach is to ignore the region above the TOA entirely (effectively treating it as a vacuum), but this produces discontinuous atmospheric profiles that can significantly deform the radiance profiles.

A better approach was developed by Chapman (1931), who modeled the profile of each constituent in an atmosphere above a certain level as falling off exponentially with height at a rate governed by the scale height of the constituent. This approach remains relatively simple, but it avoids discontinuity of the model atmosphere and also has some degree of physical realism (for example, the molecular number density in an isothermal atmosphere will decay with a constant scale height to the extent that variations of gravity with altitude can be ignored). An atmospheric region that behaves in this way is commonly known as a "Chapman layer".

The L04 GSLS model contained an option to treat the atmospheric region from the TOA to infinity as a Chapman layer, following the computational strategy suggested by Fitzmaurice (1964). However, this option was erroneously disabled by a switch in the L04 GSLS model, causing the atmosphere above the TOA to be treated like a vacuum (this switch was put in place to enable comparisons with RT models that lacked a Chapman layer capability but was accidentally never disengaged at the conclusion of those studies).

In the current GSLS model, the Chapman layer routine is restored to proper functionality. Although such details have little effect, the example shown in Fig. 9 corresponds to $\theta_{T}=60^{\circ}, \phi_{T}=90^{\circ}, R=0$, and $\lambda=345 \mathrm{~nm}$ for the atmosphere used in the L04 study. Figure 9 shows that when the TOA is sufficiently high above the maximum tangent height $h$ of interest, the inclusion or exclusion of the Chapman layer 


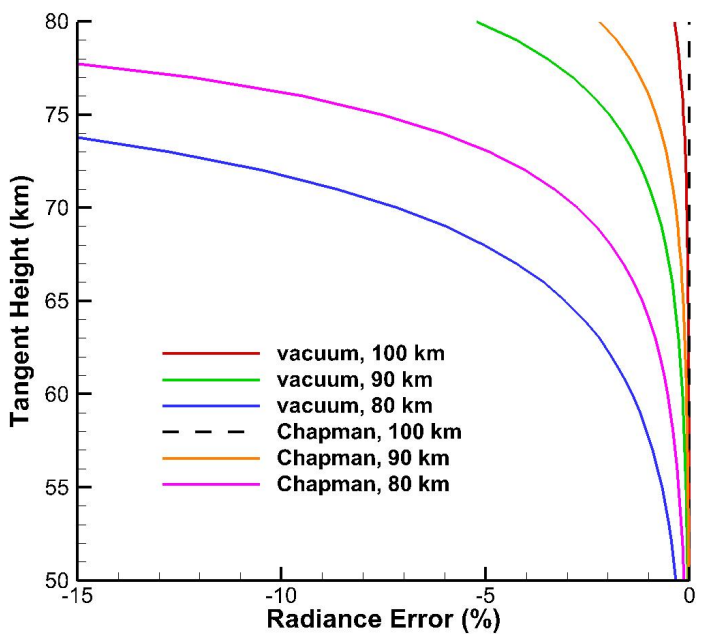

Figure 9. Total radiance error caused by changes in the TOA altitude and treatment of the atmosphere above the TOA. The reference radiance profile is calculated with TOA $=100 \mathrm{~km}$ and the Chapman layer included above the TOA. The legend indicates how the atmosphere above the TOA is handled (as a vacuum or as a Chapman layer), followed by the TOA altitude (in $\mathrm{km}$ ).

Table 6. Aerosol size distribution details for the simulated radiances shown in Fig. 10. Each size distribution is bimodal log-normal and is therefore defined by the concentration $N$, mode radius $r$, and standard deviation $\sigma$ for fine and coarse particle modes (indicated by subscript $f$ and $c$, respectively).

\begin{tabular}{lcr}
\hline & ASD20 & ASD25 \\
$N_{f}\left(\mathrm{~cm}^{-3}\right)$ & 6.57167 & 5.71833 \\
$r_{f}(\mathrm{~nm})$ & 76.55 & 51.05 \\
$\sigma_{f}$ & 1.310 & 1.43833 \\
$N_{c}\left(\mathrm{~cm}^{-3}\right)$ & 0.53642 & 0.23473 \\
$r_{c}(\mathrm{~nm})$ & 264.533 & 202.5 \\
$\sigma_{c}$ & 1.485 & 1.150 \\
\hline
\end{tabular}

has little influence on the LS radiance. For example, the TOA was set at $100 \mathrm{~km}$ in the L04 comparisons, while the radiance comparisons ended at $h=60 \mathrm{~km}$, so the lack of a Chapman layer had a negligible impact in that study. The absolute value of the percentage error in radiance due to exclusion of the Chapman layer under those conditions is $<0.02 \%$ for $h<60 \mathrm{~km},<0.07 \%$ for $h<70 \mathrm{~km}$, and $<0.5 \%$ for $h<80 \mathrm{~km}$. Including the Chapman layer rapidly becomes more consequential as the TOA and the maximum $h$ of interest move closer together: for example, the radiance error at $h=70 \mathrm{~km}$ approaches $8 \%$ when the TOA $=80 \mathrm{~km}$ and the Chapman layer is excluded but is reduced to $<3 \%$ when the $\mathrm{TOA}=80 \mathrm{~km}$ and the Chapman layer is included.

\subsection{Variable aerosol size distribution (ASD)}

Stratospheric aerosol measurement campaigns (Deshler et al., 2003) clearly demonstrate that the aerosol size distribu-

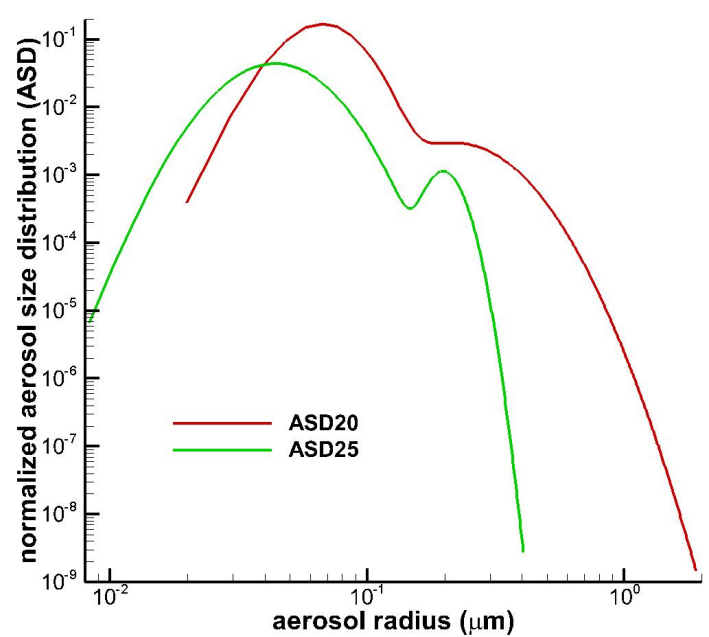

Figure 10. Illustration of the normalized aerosol size distributions (ASDs) for two bimodal log-normal stratospheric aerosol cases. The two distributions were calculated by averaging the individual ASD properties retrieved during six balloon flights over Laramie, Wyoming, during 2012 (Deshler, 2013). The details of the ASD20 size distribution (derived from observations at altitude $z=20 \mathrm{~km}$ ) and ASD25 size distribution (derived from observations at $z=$ $25 \mathrm{~km}$ ) are given in Table 6.

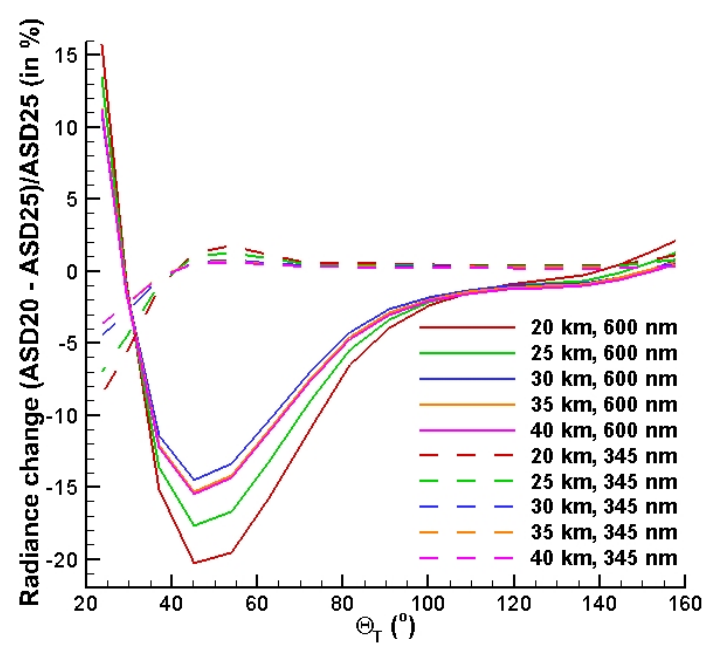

Figure 11. Comparison of TS radiances calculated at $\lambda=325 \mathrm{~nm}$ and $600 \mathrm{~nm}$ for the two ASDs plotted in Fig. 10, holding all other quantities (including aerosol extinction coefficient) constant. The legend indicates $\lambda(\mathrm{nm})$ and $h(\mathrm{~km})$.

tion varies significantly with altitude (typically with smaller particles at higher altitudes). The L04 GSLS model was incapable of including such variation - the same aerosol ASD and optical properties (refractive indices) were used for all aerosols in the atmosphere regardless of altitude. The current GSLS model has been updated to allow the aerosol ASD and optical properties (and therefore the aerosol phase function) to vary with altitude. 
Figure 10 shows two bimodal log-normal ASDs that were created by averaging the individual ASD properties (aerosol concentration $N$, aerosol mode radius $r$, and aerosol mode standard deviation $\sigma$ ) retrieved during six balloon flights over Laramie, Wyoming, during 2012 (Deshler, 2013). The red line indicates the resulting ASD for altitude $z=20 \mathrm{~km}$ (ASD20), while the green line indicates the ASD at $z=$ $25 \mathrm{~km}$ (ASD25). The details of these two size distributions are given in Table 6.

As a crude indication of the significance of the ASD for LS radiances, Fig. 11 compares the TS radiances calculated when the entire atmosphere uses ASD20 to the TS radiances calculated when the entire atmosphere uses ASD25. The aerosol phase function differs between the two cases, but other quantities (such as the aerosol extinction coefficient) are fixed in each case. The magnitude of the radiance sensitivity to ASD at $600 \mathrm{~nm}$ can be as large as $20 \%$, suggesting that an oversimplified portrayal of the stratospheric ASD (e.g., excluding the phase function variation with altitude) may be a significant source of aerosol extinction retrieval error. Figure 11 also shows that the sensitivity at $345 \mathrm{~nm}$ is less than at $600 \mathrm{~nm}$ due to the predominance of Rayleigh scattering at the UV wavelength. The magnitude of the radiance difference at $345 \mathrm{~nm}$ between the ASD20 and ASD25 cases is $<9 \%$ everywhere and $<2 \%$ for $\Theta_{T}<40^{\circ}$.

\section{Conclusions}

The GSLS RT model has been updated relative to the L04 version, significantly improving the calculated limb scattered radiances. The single-scattered radiance error now is generally $<0.3 \%$ and the total radiance error has been reduced to the $1-3 \%$ level. These accuracy gains arise primarily from improving the treatment of extinction variation within atmospheric layers (described in Sect. 4.1) and adding calculations of the multiple-scattering source function at multiple solar zenith angles along the limb scattering line of sight (described in Sect. 4.2). The required number of solar zenith angles for accurate radiance calculations is shown to be relatively modest: three or fewer are sufficient for most lines of sight, and six are sufficient for all cases except twilight conditions. The ability to model surface reflectivity variations and multiple aerosol types within the model atmosphere has also been added to the GSLS model, enabling more realistic model atmospheres to be simulated. As shown in previous studies, the OMPS LP retrieval algorithms are resilient, tolerating numerous radiative transfer model approximations without significantly changing the retrieved profiles, but these model improvements will make the GSLS model more useful for understanding OMPS LP radiance residuals. Proposed future work includes fully implementing the ability to vary the atmospheric profiles along the limb line of sight. 


\section{Appendix A}

Previous work by Herman et al. (1994) and Herman et al. (1995) describes an RT model that calculates the MS along a chosen zenith in a spherical atmosphere by employing a conical boundary at which the incident radiation from the surrounding atmosphere is estimated. The axis of the cone coincides with the zenith of interest, with cone vertex angle $=1^{\circ}$. The MS radiance is calculated at all desired levels and in all desired directions by integrating along the LOS from the conical boundary to the zenith of interest. The GSLS RT model uses a simplified, pseudo-spherical method to estimate the incident radiance on the conical boundary. In the L04 version of GSLS, a single cone was defined surrounding the zenith that intersected the TP. The resulting MS source functions were then integrated along the entire limb LOS to estimate the limb MS radiance, as described in Appendix A of L04.

The current GSLS model introduces $N_{\theta}$ MS zeniths of interest along the LOS, as described in Sect. 4.2. The MS calculation routines are then called $N_{\theta}$ times, calculating the MS source functions independently at $N_{\theta}$ zeniths along the LOS. Note that the MS radiances calculated at one zenith do not explicitly feed into the calculation of the MS at adjacent zeniths as occurs in the real atmosphere. Instead, the approximate calculation of MS source functions that were done just once (at $\theta_{T}, \phi_{T}$ ) in the L04 GSLS model is now done independently at $N_{\theta}$ values of solar zenith angle and relative azimuth angle along the LOS. The current GSLS model therefore uses a form of the "independent pixel approximation" (Cahalan et al., 1994) rather than fully modeling the MS radiance in the spherical atmosphere.

To estimate the MS at points for which the value was not explicitly calculated, the MS source function at a particular altitude and direction is assumed to vary with the cosine of the solar zenith angle between the two bounding values. This approximation is fairly crude, but the resulting MS radiances improved by an acceptable amount with modest additional computational effort, as shown in Figs. $5 \mathrm{~b}$ and 7 and Table 4.

\section{Appendix B}

As noted in Eq. (1), the optical path length through a layer $\tau$ is given by the integral of the extinction coefficient $\beta$ along the LOS through the layer. If we assume that $\beta$ varies linearly with height between the endpoints of the path (emulating the method used by the Siro model as described in L04), then the integral shown in Eq. (1) has an analytic solution that can be obtained from standard tables of integrals (for both tangent and non-tangent paths). We will use the subscripts 0 and 1 to indicate the values of various quantities at the endpoints of the LOS, corresponding to the lower and upper limits of the integral in Eq. (1), respectively. For a spherical Earth, $r_{0}$ and $r_{1}$ indicate the distances from the center of the Earth to endpoints 0 and 1 , respectively (for example, $r_{0}=O Q$ and

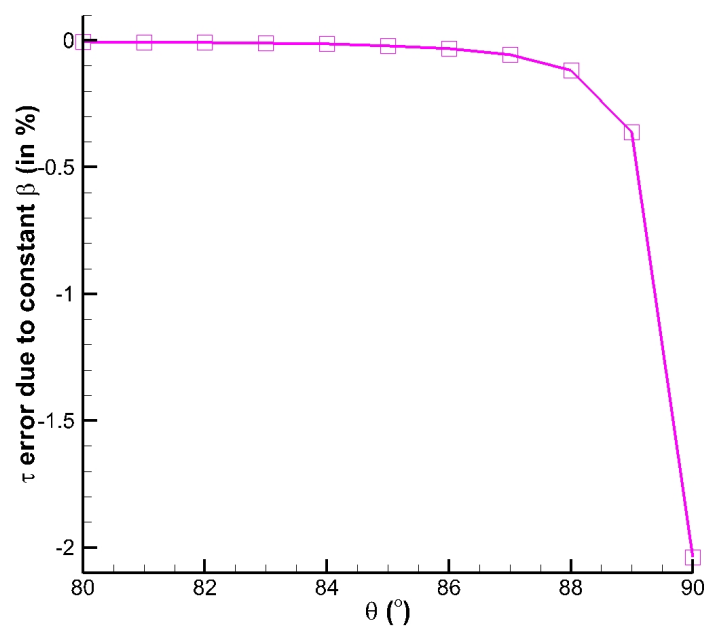

Figure B1. Percentage difference between the value of $\tau$ obtained from Eq. (2) and the value of $\tau$ obtained from Eq. (B8) as a function of $\theta$. The LOS has $h=60 \mathrm{~km}$ in this example.

$r_{1}=O T$ in Fig. 2). Similarly, $\beta_{0}$ and $\beta_{1}$ represent the values of the extinction coefficient at endpoints 0 and 1. Finally, the angle between the LOS (in the direction the radiation is traveling) and the zenith line that intersects the LOS at $r_{0}$ (outwards from the center of the Earth) is denoted by $\theta$ (i.e., the angle $\theta_{Q}$ in Fig. 2).

If we assume that $\beta$ is a linear function of altitude (or $r$ ) within the relevant layer, then the following expressions obviously follow:

$\beta(r)=A r+B$

where

$A=\left(\beta_{0}-\beta_{1}\right) /\left(r_{0}-r_{1}\right)$

$B=\beta_{0}-A r_{0}$.

Substituting Eq. (B1) into Eq. (1) yields

$\tau=\int_{0}^{s}\left(A r^{\prime}+B\right) d s^{\prime}=B s+A \int_{0}^{s} r^{\prime} d s^{\prime}$.

To evaluate Eq. (B3), the variation of $r^{\prime}$ along the LOS from $r_{0}$ to $r_{1}$ must be characterized. This can be determined from the law of cosines as

$$
\begin{aligned}
& r^{\prime}=\sqrt{s^{\prime 2}-2 s^{\prime} r_{0} \cos (\pi-\theta)+r_{0}^{2}}=\sqrt{s^{\prime 2}+2 s^{\prime} r_{0} \cos \theta+r_{0}^{2}} \\
& r^{\prime}=\sqrt{c s^{\prime 2}+b s^{\prime}+a}
\end{aligned}
$$

As implied in Eq. (B4), $a=r_{0}^{2}, b=2 r_{0} \cos \theta$, and $c=1$. Substituting Eq. (B4) into Eq. (B3) yields an integral that can be evaluated analytically based on CRC (1984), equations 242 and 237 , using the shorthand $k^{-1}=\left(4 a c-b^{2}\right) / 4 c=$ 
$r_{0}^{2} \sin ^{2} \theta:$

$$
\begin{aligned}
& \int_{0}^{s} \mathrm{~d} s^{\prime} \sqrt{c s^{\prime 2}+b s^{\prime}+a} \\
& =\left\{\frac{2 c s^{\prime}+b}{4 c} \sqrt{c s^{\prime 2}+b s^{\prime}+a}+\frac{k^{-1}}{2 \sqrt{c}}\right. \\
& \left.\log \left[2 \sqrt{c\left(c s^{\prime 2}+b s^{\prime}+a\right)}+2 c s^{\prime}+b\right]\right\}_{0}^{s} .
\end{aligned}
$$

Substituting for $a, b, c$, and $k^{-1}$ and evaluating Eq. (B5) yields

$$
\begin{aligned}
& \int_{0}^{s} \mathrm{~d} s^{\prime} \sqrt{c s^{\prime 2}+b s^{\prime}+a}=\frac{1}{2}\left\{\left(s+r_{0} \cos \theta\right)\right. \\
& \left.\sqrt{s^{2}+2 r_{0} s \cos \theta+r_{0}^{2}}-r_{0}^{2} \cos \theta\right\} \\
& +\frac{1}{2}\left\{r_{0}^{2} \sin ^{2} \theta \log \left[2\left(\sqrt{s^{2}+2 r_{0} s \cos \theta+r_{0}^{2}}+s+r_{0} \cos \theta\right)\right]\right. \\
& \left.-r_{0}^{2} \sin ^{2} \theta \log \left[2\left(r_{0}+r_{0} \cos \theta\right)\right]\right\} .
\end{aligned}
$$

This can be further simplified by noting (again from the law of cosines) that $r_{1}=\sqrt{s^{2}+2 r_{0} s \cos \theta+r_{0}^{2}}$. With that substitution and some rearrangement, Eq. (B6) becomes

$$
\begin{aligned}
& \int_{0}^{s} d s^{\prime} \sqrt{c s^{\prime 2}+b s^{\prime}+a}=\frac{1}{2}\left\{s r_{1}+r_{0}\left(r_{1}-r_{0}\right) \cos \theta\right. \\
& \left.+r_{0}^{2} \sin ^{2} \theta \log \left[\frac{r_{1}+s+r_{0} \cos \theta}{r_{0}(1+\cos \theta)}\right]\right\} .
\end{aligned}
$$

So the final solution for $\tau$, combining Eqs. (B7) and (B3), becomes

$$
\begin{aligned}
& \tau=B s+\frac{A}{2}\left\{s r_{1}+r_{0}\left(r_{1}-r_{0}\right) \cos \theta+r_{0}^{2} \sin ^{2} \theta\right. \\
& \left.\log \left[\frac{r_{1}+s+r_{0} \cos \theta}{r_{0}(1+\cos \theta)}\right]\right\},
\end{aligned}
$$

which can be evaluated using the definitions of $A$ and $B$ given in Eq. (B2). The expression Eq. (B8) has the proper limiting behavior: when $\theta=0^{\circ}$, Eq. (B8) reduces to Eq. (2). This corresponds to the case of radiation propagating radially outward from the center of the Earth; thus $r_{1}=r_{0}+s$ and Eq. (1) yields the result given in Eq. (2) when $\tau$ is a linear function of $r$. When $\theta=180^{\circ}$, radiation propagates radially inward, $r_{1}=r_{0}-s$, and the argument of the logarithm in Eq. (B8) becomes indeterminate. Equation (B8) therefore cannot be used directly as an algorithm when $\theta=180^{\circ}$, but it remains true that the value of $\tau$ should approach the expression given in Eq. (2) as the path becomes vertical, so the failure of Eq. (B8) for the $\theta=180^{\circ}$ case can be easily avoided by reverting to Eq. (2) when $\theta \longrightarrow 180^{\circ}$.

The far more consequential case is $\theta \longrightarrow 90^{\circ}$ (nearlytangent paths): the value of $\tau$ obtained from Eq. (2) always represents an underestimate and can be over $2 \%$ smaller than $\tau$ obtained from (Eq. B8), as shown in Fig. B1. As alluded to in Sect. 4.1, the L04 GSLS model made a partial correction for the error caused by using Eq. (2) to estimate $\tau$ in the tangent layer by estimating the variation of $\beta$ within the tangent layer based on the profile of $\beta$ in adjacent layers. However, the L04 GSLS tangent layer approximation was imperfect, and no correction was made in the L04 GSLS model for $\tau$ in nearly tangent layers, so Eq. (B8) represents a clear improvement in the current GSLS model. 


\section{The Supplement related to this article is available online at doi:10.5194/acp-15-3007-2015-supplement.}

Acknowledgements. The majority of the material presented in this paper first appeared as a poster at the 7th Atmospheric Limb Conference, which was hosted by the University of Bremen, Germany, in June 2013. This research was supported by NASA GSFC through SSAI subcontract 21205-12-043. The authors thank NASA and NOAA for supporting limb scattering research and particularly recognize Didier Rault for years of leadership developing the OMPS LP algorithms. Larry Thomason and Terry Deshler shared helpful insights into the stratospheric aerosol problem. The NASA, SSAI, and NOAA OMPS teams supported this research and contributed many useful discussions, including Ghassan Taha, Larry Flynn, Zhong Chen, Philippe Xu, Tong Zhu, Steve Taylor, Matt DeLand, Nick Gorkavyi, Al Fleig, Jack Larsen, Mike Linda, Leslie Moy, and Peter Hall. Several Hampton University students contributed to studies that have improved the GSLS model, including Daryl Ludy, Simone Hyater-Adams, Ricardo Uribe, Curtis Driver, Jonathan Geasey, Nicholas Carletta, and Darel Davidson. Two anonymous reviewers provided valuable comments that improved this paper. We appreciate the OSIRIS, SCIAMACHY, SAGE II, SAGE III, and University of Wyoming measurement teams for maintaining and sharing their high-quality data sets. Finally, we thank Alexei Rozanov and the University of Bremen team for enabling remote participation in the 7th Atmospheric Limb Conference.

Edited by: R. Ebinghaus

\section{References}

Bovensmann, H., Burrows, J. P., Buchwitz, M., Frerick, J., Noël, S., Rozanov, V. V., Chance, K. V., and Goede, A. P. H.: SCIAMACHY: mission objectives and measurement modes, J. Atmos. Sci., 56, 127-150, 1999

Bourassa, A. E., Degenstein, D. A., and Llewellyn, E. J.: SASKTRAN: a spherical geometry radiative transfer code for efficient estimation of limb scattered sunlight, J. Quant. Spectrosc. Rad., 109, 57-73, 2008.

Cahalan, R. F., Ridgeway, W., Wiscombe, W. J., Bell, T. L., and Snider, J. B.: The albedo of fractal stratocumulus clouds, J. Atmos. Sci., 51, 2434-2455, 1994.

Chapman, S.: The absorption and dissociative or ionizing effect of monochromatic radiation in an atmosphere on a rotating Earth. Part II. Grazing incidence, Proc. Phys. Soc., 43, 483-501, 1931.

Coulson, K. L., Dave, J. V., and Sekera, Z.: Tables related to radiation emerging from a planetary atmosphere, U. California, Berkeley, 1960.

CRC: Standard Mathematical Tables, 27. edn., edited by: Beyer, W. H., CRC Press, Inc., Boca Raton, Florida, 1984

Deshler, T.: University of Wyoming stratospheric aerosol size distributions, http://www-das.uwyo.edu/ deshler/Data/Aer_Meas_ Wy_read_me.htm, last access: February 2013.
Deshler, T., Hervig, M. E., Hoffman, D. J., Rosen, J. M., and Liley, J. B.: Thirty years of in situ stratospheric aerosol size distribution measurements from Laramie, Wyoming $\left(41^{\circ} \mathrm{N}\right)$, using balloon-borne instruments, J. Geophys. Res., 108, 4167, doi:10.1029/2002JD002514, 2003.

Deutschmann, T., Beirle, S., Frieß, U., Grzegorski, M., Kern, C., Kritten, L., Platt, U., Prados-Román, C., Pukite, J., Wagner, T., Werner, B., and Pfeilsticker, K.: The Monte Carlo atmospheric radiative transfer model McArtim: Introduction and validation of Jacobians and 3D features, J. Quant. Spec. Rad. Trans., doi:10.1016/j.jqsrt.2010.12.009, 2011.

Fitzmaurice, J. A.: Simplification of the Chapman function for atmospheric attenuation, Appl. Optics, 3, p. 640, 1964.

Flittner, D. E., Bhartia, P. K., and Herman, B. M.: $O_{3}$ profiles retrieved from limb scatter measurements: theory, Geophys. Res. Lett., 27, 2601-2604, 2000.

Flynn, L. E., Seftor, C. J., Larsen, J. C., and Xu, P.: The Ozone Mapping and Profiler Suite, Earth Science Satellite Remote Sensing, Volume 1: Science and Instruments, edited by: Qu, J., Gao, W., Kafatos, M., Murphy, R. E., and Salomonson, V. V., 279-295, Tsinghua University Press, Beijing and Springer, Berlin Heidelberg New York, doi:10.1007/978-3-540-37293-6, 2006.

Griffioen, E. and Oikarinen, L.: LIMBTRAN: a pseudo threedimensional radiative transfer model for the limb-viewing imager OSIRIS on the ODIN satellite, J. Geophys. Res., 105, 29717 29730, 2000.

Herman, B. M., Caudill, T. R., Flittner, D. E., Thome, K. J., and Ben-David, A.: Comparison of the Gauss-Seidel spherical polarized radiative transfer code with other radiative transfer codes, Appl. Optics, 34, 4563-4572, 1995.

Herman, B. M., Ben-David, A., and Thome, K. J.: Numerical techniques for solving the radiative transfer equation for a spherical shell atmosphere, Appl. Optics, 33, 1760-1770, 1994.

Jaross, G., Bhartia, P. K., Chen, G., Kowitt, M., Haken, M., Chen, Z., Xu, P., Warner, J., and Kelly, T.: OMPS Limb Profiler instrument performance assessment, J. Geophys. Res., 119, doi:10.1002/2013JD020482, 2014.

Kyrölä, E., Tamminen, J., Leppelmeier, G. W., Sofieva, V., Hassinen, S., Bertaux, J. L., Hauchecorne, A., Dalaudier, F., Cot, C., Korablev, O., Fanton d'Andon, O., Barrot, G., Mangin, A., Théodore, B., Guirlet, M., Etanchaud, F., Snoeij, P., Koopman, R., Saavedra, L., Fraisse, R., Fussen, D., and Vanhellemont, F.: GOMS on ENVISAT: an overview, Adv. Space Res., 33, 10201028, doi:10.1016/S0273-1177(03)00590-8, 2004.

Llewellyn, E. J., Lloyd, N. D., Degenstein, D. A., Gattinger, R. L., Petelina, S. V., Bourassa, A. E., Wiensz, J. T., Ivanov, E. V., McDade, I. C., Solheim, B. H., McConnell, J. C., Haley, C. S., von Savigny, C., Sioris, C. E., McLinden, C. A., Griffioen, E., Kaminski, J., Evans, W. F., Puckrin, E., Strong, K., Wehrle, V., Hum, R. H., Kendall, D. J. W., Matsushita, J., Murtagh, D. P., 5 Brohede, S., Stegman, J., Witt, G., Barnes, G., Payne, W. F., Piché, L., Smith, K., Warshaw, G., Deslauniers, D.-L., Marchand, P., Richardson, E. H., King, R. A., Wevers, I., McCreath, W., Kyrölä, E., Oikarinen, L., Leppelmeier, G. W., Auvinen, H., Mégie, G., Hauchecorne, A., Lefèvre, F., de La Nöe, J., Ricaud, P., Frisk, U., Sjoberg, F., von Schéele, F., and Nordh, L.: The OSIRIS instrument on the Odin spacecraft, Can. J. Phys., 82, 411-422, 2004. 
Loughman, R. P., Griffioen, E., Oikarinen, L., Postylyakov, O. V., Rozanov, A., Flittner, D. E., and Rault, D. F.: Comparison of radiative transfer models for limb-viewing scattered sunlight measurements, J. Geophys. Res., 109, D06303, doi:10.1029/2003JD003854, 2004.

Loughman, R. P., Flittner, D. E., Herman, B. M., Bhartia, P. K., Hilsenrath, E., and McPeters, R. D.: Description and sensitivity analysis of a limb scattering ozone retrieval algorithm, J. Geophys. Res., 110, doi:10.1029/2004JD005429, 2005.

McLinden, C. A. and Bourassa, A. E.: A systematic error in planeparallel radiative transfer calculations, J. Atmos. Sci., 67, 16951699, 2010.

McLinden, C. A., McConnell, J. C., Griffioen, E., and McElroy, C. T.: A vector radiative transfer model for the Odin/OSIRIS project, Can. J. Phys., 80, 375-393, 2002.

McPeters, R. D., Janz, S. J., Hilsenrath, E., Brown, T. L., Flittner, D. E., and Heath, D. F.: The retrieval of $O_{3}$ profiles from limb scatter measurements: results from the shuttle ozone limb sounding experiment, Geophys. Res. Lett., 27, 2597-2600, 2000.

Mishchenko, M. I., Lacis, A. A., and Travis, L. D.: Errors induced by the neglect of polarization in radiance calculations for Rayleigh-scattering atmospheres, J. Quant. Spectrosc. Ra., 51, 491-510, 1994.

Mount, G. H., Rusch, D. W., Noxon, J. F., Zawodny, J. M., and Barth, C. A.: Measurements of stratospheric $\mathrm{NO}_{2}$ from the SME Satellite, J. Geophys. Res., 89, 1327-1340, 1984.

Natraj, V., Li, K., and Yung, Y. L.: Rayleigh scattering in planetary atmospheres: corrected tables through accurate computation of X and Y functions, Astrophys. J., 691, 1909-1920, 2009.

Oikarinen, L., Sihvola, E., and Kyrölä, E.: Multiple scattering radiance in limb-viewing geometry, J. Geophys. Res., 104, 3126131274, 1999.

Postylyakov, O. V.: Radiative transfer model MCC++ with evaluation of weighting functions in spherical atmosphere for usage in retrieval algorithms, Adv. Space Res., 34, 721-726, 2004.

Premuda, M., Palazzi, E., Ravegnani, F., Bortoli, D., Masieri, S., and Giovanelli, G.: MOCRA: a Monte Carlo code for the simulation of radiative transfer in the atmosphere, Opt. Express, 20, 7973-7993, 2012.

Rault, D. F., Ozone profile retrieval from SAGE III limb scattering measurements, J. Geophys. Res., 110, doi:10.1029/2004JD004970, 2005.
Rault, D. F. and Taha, G.: Validation of ozone profiles retrieved from Stratospheric Aerosol and Gas Experiment III limb scatter measurements, J. Geophys. Res., 112, doi:10.1029/2006JD007679, 2007.

Rault, D. and Loughman, R. P.: The OMPS limb profiler environmental data record algorithm theoretical basis document and expected performance, IEEE T. Geosci. Remote, 51, doi:10.1109/TGRS.2012.2213093, 2013.

Rozanov, V. V., Rozanov, A., Kokhanovsky, A. A., and Burrows, J. P.: Radiative transfer through terrestrial atmosphere and ocean: software package SCIATRAN, J. Quant. Spectrosc. Radiat. Transfer, 133, 13-71, 2014.

Rozanov, A., Rozanov, V., and Burrows, J. P.: A numerical radiative transfer model for a spherical planetary atmosphere: Combined differential-integral approach involving the picard iterative approximation, J. Quant. Spectrosc. Radiat. Transfer, 69, 491-512, 2001.

Rusch, D. W., Mount, G. H., Barth, C. A., Thomas, R. J., and Callan, M. T.: Solar mesosphere explorer ultraviolet spectrometer: measurements of ozone in the 1.0-0.1 mbar region, J. Geophys. Res., 89, 11677-11687, 1984.

Spada, F., Krol, M. C., and Stammes, P.: McSCIA: application of the Equivalence Theorem in a Monte Carlo radiative transfer model for spherical shell atmospheres, Atmos. Chem. Phys., 6, 48234842, doi:10.5194/acp-6-4823-2006, 2006.

Taha, G., Jaross, G., Fussen, D., Vanhellemont, F., Kyrölä, E., and McPeters, R. D.: Ozone profile retrieval from GOMOS limb scattering measurements, J. Geophys. Res., 113, D23307, doi:10.1029/2007JD009409, 2008.

Taha, G., Rault, D. F., Loughman, R. P., Bourassa, A. E., and von Savigny, C.: SCIAMACHY stratospheric aerosol extinction profile retrieval using the OMPS/LP algorithm, Atmos. Meas. Tech., 4, 547-556, doi:10.5194/amt-4-547-2011, 2011.

Whitney, C. K.: The DART Method, Standard Procedures to Compute Radiative Transfer in a Scattering Atmosphere, J. Lenoble, Ed., Radiation Commission, International Association of Meteorology and Atmospheric Physics, I. U. G. G., published by National Center for Atmospheric Research, Boulder, Colorado, 80 83, 1977. 